\title{
A novel porcine model reproduces human oculocutaneous albinism type II
}

\author{
Ying Zhang ${ }^{1,2}$, Qianlong Hong ${ }^{3}$, Chunwei Cao ${ }^{1,2}$, Lizhu Yang ${ }^{4}$, Yongshun Li ${ }^{1,2}$, Tang Hai ${ }^{1,2}$, Hongyong Zhang ${ }^{1,2}$, \\ Qi Zhou $\mathbb{1}^{1,2}$, Ruifang Sui ${ }^{4}$ and Jianguo Zhao ${ }^{1,2}$
}

Dear Editor,

Oculocutaneous albinism (OCA) represents a genetically heterogeneous group of disorders characterized by absent or reduced pigmentation of the skin, hair, and eyes from the time of birth ${ }^{1}$. OCA type II (OCA2) is one of the most common type of the disorder, and accounts for $30 \%$ of cases worldwide ${ }^{2}$. However, no effective treatments or medicines exist for curing this disease currently, thus it is necessary to generate animal models for evaluating novel medicines or developing novel therapeutic interventions for the clinic. Previous studies have reported several mouse models for OCA2. However, in addition to showing some of the clinical manifestations of OCA2, several mutant murine strains are accompanied by other abnormalities, including decreased neonatal viability, increased prenatal lethality, reproductive and neurological disorders, and incidence of cleft palate ${ }^{3,4}$. It suggests that mice may not fully recapitulate the OCA phenotype, thus highlighting the need for a more suitable animal model. Here, we created a porcine model of OCA2 to bridge the gap between human clinical cases and rodent animal models, and the porcine model displays overt hypopigmentation in eyes and hair follicles similar to those observed in OCA2 patients and lacked other apparent abnormities.

Compared to the coat color pattern of a unique two-end black, the mutant Bama miniature pigs in the Z0015 line exhibiting a two-end diluted brown coat color were identified from an ethylnitrosourea (ENU) mutagenesis $\operatorname{program}^{5}$ (Fig. 1a). Pink eyes also accompanied the

\footnotetext{
Correspondence: Ruifang Sui (hrfsui@163.com) or Jianguo Zhao (zhaojg@ioz.ac $\mathrm{cn})$

${ }^{1}$ State Key Laboratory of Stem Cell and Reproductive Biology, Institute of

Zoology, Chinese Academy of Sciences, 100101 Beijing, China

${ }^{2}$ University of Chinese Academy of Sciences, 100049 Beijing, China

Full list of author information is available at the end of the article.
}

dilution of coat color in the mutant pigs (Fig. 1b); these unique features were similar to those of OCA in patients ${ }^{6}$. In the mutant pig line, a G1 boar (Z0015) was backcrossed to four G2 sows that produced 65 G3 offspring. Of the progeny, 21.5\% (8 males and 6 females) exhibited the mutant phenotype in an autosomal recessive inheritance pattern (Fig. 1c, d).

To identify the causative gene in the Z0015 line, we performed a family-based genome-wide linkage study (GWLS) to map the chromosome regions co-segregating with the mutant phenotypes. A significant linkage peak was identified between $46 \mathrm{Mb}$ and $66 \mathrm{Mb}$ on chromosome 15 (LOD > 2) containing 30 annotated genes (Fig. 1e). Of these candidate genes, OCA2 was of particular interest due to pathogenic mutations in the $O C A 2$ gene are known to cause OCA2, one subtype of $\mathrm{OCA}^{7}$. Using genomic DNA, 23 coding exons flanked by introns of the porcine OCA2 gene (GenBank ID: 397171) were screened. The results showed a single missense mutation c. $2228 \mathrm{C}>\mathrm{T}$ that resulted in a serine transition to leucine (S743L, hereafter referred to as $O C A 2^{S 743 L / S 743 L}$ ) in exon 21 (Fig. 1f).

To further investigate the association between the altered allele and the mutant phenotype, we genotyped the c. $2228 \mathrm{C}>\mathrm{T}$ mutation in various pig breeds and the results revealed that the $\mathrm{T} / \mathrm{T}$ homozygous mutant genotype was only found in the affected pigs from the Z0015 line but not in the other Bama miniature pigs or other breeds (Supplementary Table S1). Together, the above results suggest that the c. $2228 \mathrm{C}>\mathrm{T}$ mutation in OCA2 co-segregated with the OCA phenotype and is the causative mutation in this mutant line. In addition, the S743L mutation in OCA2 was not found in the pig SNPs database, suggesting that S743L was induced by ENU mutagenesis.

The S743L mutation is located in the predicted transmembrane domain 10 of the porcine OCA2 protein and is evolutionarily conserved among distinct mammals 


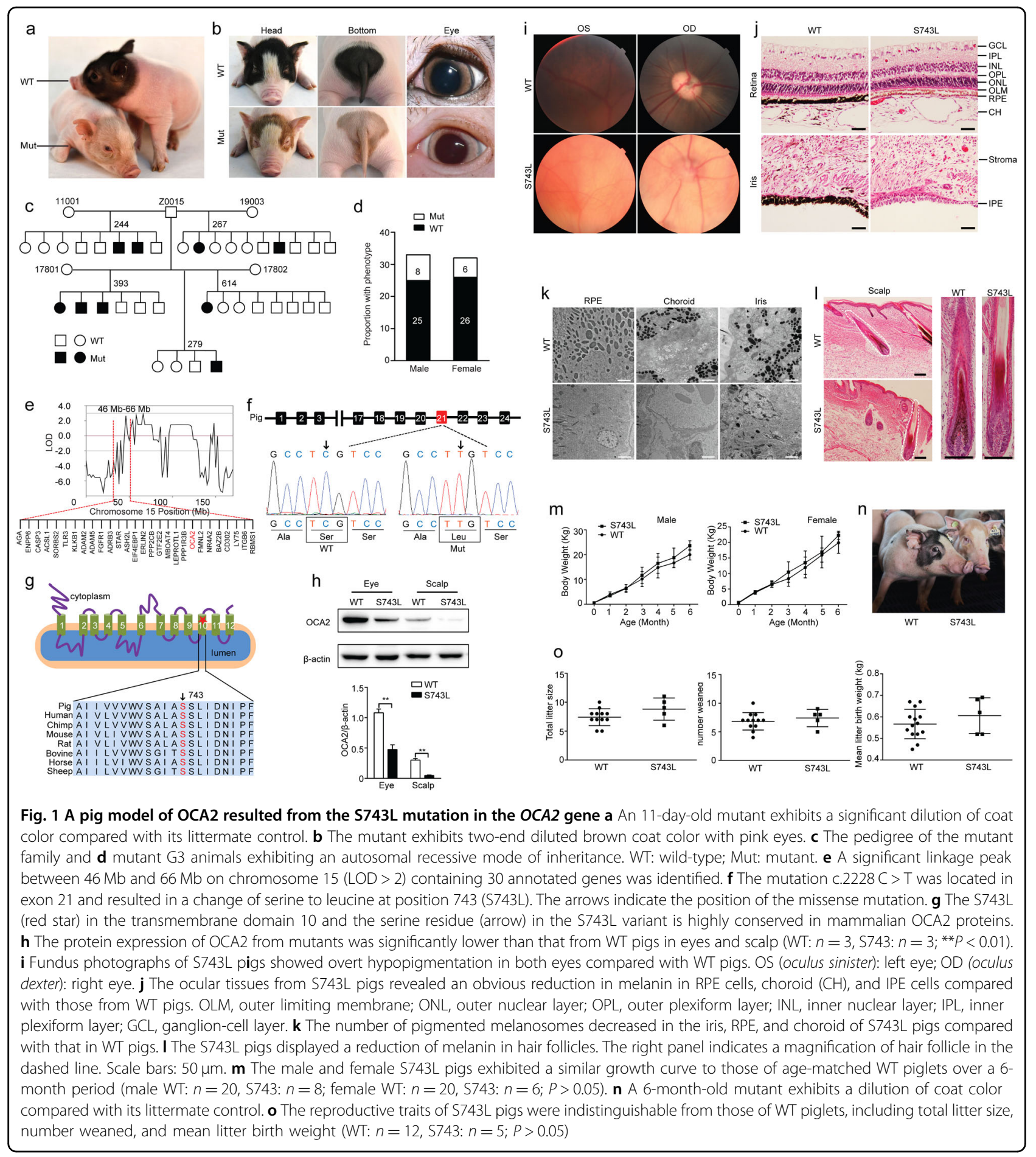

(Fig. 1g), indicating its vital role within this domain for the function of OCA2. The S743L mutation did not alter $O C A 2$ expression at the transcript level (Supplementary Fig. S1); however, the protein expression from mutant pigs was significantly lower than that from WT pigs in eyes and scalp (Fig. 1h). Furthermore, supporting our discovery, the patient manifests a typical moderate OCA2 phenotype was found carrying a compound heterozygote for two missense substitutions, S736L (orthologous mutation to porcine $\mathrm{S} 743 \mathrm{~L}$ ) and $\mathrm{G}_{27 \mathrm{R}^{8}}$. This suggests that S743L-mutant pigs are suitable for modeling human OCA2 due to uniform genetics.

We further analyzed the morphological and pathological phenotypes in the eyes and skin of the OCA $2^{\text {S743L/S743L }}$ 
pigs. Fundus photographs showed overt hypopigmentation in both eyes from the mutants compared with the unaffected controls (Fig. 1i). The ocular tissues from mutant pigs revealed an obvious reduction of melanin in the retinal pigment epithelium (RPE) cells and choroid $(\mathrm{CH})$, and an almost complete lack of melanin in the iris pigment epithelium (IPE) cells (Fig. 1j). Furthermore, iris, $\mathrm{RPE}$, and $\mathrm{CH}$ of mutants displayed an evident decrease in the number of the pigmented melanosomes compared to those of the WTs, which was consistent with the histopathological findings (Fig. 1k). Electroretinograms (ERGs) were used to assess changes in retinal function, and the results showed that no significant functional differences existed between mutant and WT pigs (Supplementary Fig. S2). In addition, the mutants also exhibited decreased levels of melanin in scalp hair follicles compared with those from WT pigs (Fig. 11). Together, these phenotypes manifest the clinical features of human OCA2 ${ }^{9}$.

To investigate whether the $5743 \mathrm{~L}$ mutation induced growth or fertility aberrations in $O C A 2^{\text {S743L/S743L }}$ pigs, we characterized the growth and reproductive traits. The growth curve revealed that the body weight of $O C A 2^{S 743 L /}$ ${ }_{S 743 L}$ piglets was similar to that of the age-matched WT piglets over a period of 6 months (Fig. $1 \mathrm{~m}$ ). The adult mutant pigs showed a consistent dilution brown coat color and pink eyes, and their physique was similar to that of WT pigs (Fig. 1n). At 6 months or greater, OCA $2^{S 743 L /}$ ${ }^{S 743 L}$ males were mated with $O C A 2^{S 743 L / S 743 L}$ females and shown to be fertile. The reproductive traits of $O C A 2^{S 743 L /}$ ${ }^{S 743 L}$ pigs were indistinguishable from those of WT piglets (Fig. 1o). In addition, the profiles of blood physiological and biochemical parameters of $O C A 2^{S 743 L / S 743 L}$ pigs at 3 months were consistent with those of age-matched WT pigs (Supplementary Fig. S3). Our results indicate that while the $O C A 2^{5743 L / S 743 L}$ mutation resulted in OCA disease phenotype but did not affect growth, reproduction, and levels of blood physiology and biochemistry.

In summary, we identified a first pig model of OCA2 resulted from the $5743 \mathrm{~L}$ mutation in the OCA2 gene. Furthermore, the S743L mutation, which is orthologous to the human S736L mutation, results solely in pigmentation-related defects without other apparent abnormities similar to those observed in OCA2 patients. Taken together, our findings suggested that $O C A 2^{S 743 L /}$ $S 743 L$ pigs could be utilized as an appropriate preclinical model in which to test human-size interventional devices and optimize candidate therapies before advancing to clinical trials, thus accelerating the development of effective applications for OCA patients.

\section{Acknowledgements}

We thank Pengyan Xia and Guopeng Wang from the State Key Laboratory of Membrane Biology, Institute of Zoology, Chinese Academy of Sciences for their technical assistance. This work was supported by the Strategic Priority Research Program of the Chinese Academy of Sciences (XDA16030304), the National Natural Science Foundation of China (31801031, 81671274, 31272440, and 81873687), the CAMS Innovation Fund for Medical Sciences (CIFMS 201612M-1-002) and the National Transgenic Project of China (2016ZX08009003006-007).

\section{Author details \\ ${ }^{1}$ State Key Laboratory of Stem Cell and Reproductive Biology, Institute of Zoology, Chinese Academy of Sciences, 100101 Beijing, China. ${ }^{2}$ University of Chinese Academy of Sciences, 100049 Beijing, China. ${ }^{3}$ School of Life Sciences, Anhui University, 230601 Hefei, China. ${ }^{4}$ Department of Ophthalmology, Peking Union Medical College Hospital, Peking Union Medical College, Chinese Academy of Medical Sciences, 100730 Beijing, China}

\section{Author contributions}

J.Z. conceived the project with input from R.S. and Q.Z.; Y.Z., Q.H., L.Y., Y.L., T.H. and H.Z. performed the experiments; Y.Z., Q.H., and C.C. analyzed the data; Y.Z. wrote the manuscript and J.Z. deeply revised the manuscript.

Conflict of interest

The authors declare that they have no conflict of interest.

\section{Publisher's note}

Springer Nature remains neutral with regard to jurisdictional claims in published maps and institutional affiliations.

Supplementary Information accompanies the paper at (https://doi.org/ 10.1038/s41421-019-0117-7).

Received: 9 June 2019 Accepted: 25 July 2019

Published online: 08 October 2019

\section{References}

1. Okulicz, J. F., Shah, R. S., Schwartz, R. A. \& Janniger, C. K. Oculocutaneous albinism. J. Eur. Acad. Dermatol. Venereol. 17, 251-256 (2003).

2. Simeonov, D. R. et al. DNA variations in oculocutaneous albinism: an updated mutation list and current outstanding issues in molecular diagnostics. Hum. Mutat. 34, 827-835, https://doi.org/10.1002/humu.22315 (2013).

3. Brilliant, M. H. The mouse $p$ (pink-eyed dilution) and human $P$ genes, oculocutaneous albinism type 2 (OCA2), and melanosomal pH. Pigment Cell Res. 14, 86-93 (2001).

4. Johnson, D. K. et al. Molecular analysis of 36 mutations at the mouse pink-eyed dilution (p) locus. Genetics 141, 1563-1571 (1995).

5. Hai, T. et al. Pilot study of large-scale production of mutant pigs by ENU mutagenesis. Elife 6, https://doi.org/10.7554/eLife.26248 (2017).

6. Gronskov, K., Ek, J. \& Brondum-Nielsen, K. Oculocutaneous albinism. Orphanet J. Rare Dis. 2, 43 (2007).

7. Gao, J. et al. Retrospective analysis in oculocutaneous albinism patients for the $2.7 \mathrm{~kb}$ deletion in the OCA2 gene revealed a co-segregation of the controversial variant, p.R305W. Cell Biosci. 7, 22 (2017).

8. Spritz, R. A. et al. Novel mutations of the P gene in type II oculocutaneous albinism (OCA2). Hum. Mutat. 10, 175-177 (1997).

9. Manga, P., Kromberg, J., Turner, A., Jenkins, T. \& Ramsay, M. In Southern Africa, brown oculocutaneous albinism (BOCA) maps to the OCA2 locus on chromosome 15q: P-gene mutations identified. Am. J. Hum. Genet. 68, 782-787 (2001). 